\title{
Neuromodulation and Eating Disorders
}

\author{
L. Gallop ${ }^{1} \cdot$ M. Flynn ${ }^{1} \cdot$ I.C. Campbell ${ }^{1} \cdot$ U. Schmidt ${ }^{1,2}$
}

Accepted: 20 November 2021 / Published online: 18 February 2022

(c) The Author(s) 2022

\begin{abstract}
Purpose of Review We review recent evidence on the use of neuromodulation for treating eating disorders (EDs), including anorexia nervosa, bulimia nervosa and binge eating disorder. We evaluate studies on (a) modern non-invasive methods of brain stimulation, such as transcranial magnetic stimulation (rTMS) and transcranial direct current stimulation (tDCS), (b) electroconvulsive therapy (ECT) and (c) more invasive techniques, including deep brain stimulation (DBS).

Recent Findings Most reports on the clinical applications of neuromodulation in EDs are limited to case studies, case series and small clinical trials. The majority have focused on severe, enduring and hard-to-treat cases of AN. In this population, data suggest that both rTMS and DBS have therapeutic potential and are safe and acceptable.

Summary High-quality clinical trials in different ED populations are needed which investigate different stimulation methods, sites and parameters, the use of neuromodulation as stand-alone and/or adjunctive treatment, as well as the mechanisms of action.
\end{abstract}

Keywords Eating disorders · Anorexia nervosa $\cdot$ Neuromodulation $\cdot$ Brain stimulation $\cdot$ Transcranial magnetic stimulation $\cdot$ Transcranial direct current stimulation

\section{Introduction}

Eating disorders (EDs) are serious psychiatric disorders with complex biological, psychological and social underpinnings [1]. Anorexia nervosa (AN) is characterised by an intense fear of weight gain and a disturbed body image which motivates severe dietary restriction or other weight loss behaviours, e.g. excessive exercise. Individuals with bulimia nervosa (BN) and binge-eating disorder (BED) experience distressing episodes of objective overeating accompanied by a sense of loss of control, with (in BN) or without (in BED) compensatory behaviours to counteract weight gain (e.g. self-induced vomiting). With mortality rates for AN as high as 5\% [2], EDs account for some of the highest rates of morbidity and mortality among mental illnesses.

This article is part of the Topical Collection on Eating Disorders

U. Schmidt

ulrike.schmidt@kcl.ac.uk

1 Section of Eating Disorders, Department of Psychological Medicine, Institute of Psychiatry, King's College London, Psychology \& Neuroscience, London, UK

2 South London and Maudsley NHS Foundation Trust, Maudsley Hospital, London, UK
Clinical guidelines recommend a range of ED focused psychological therapies as first-line treatments for EDs [3]. However, outcomes from the best available psychotherapies for EDs are suboptimal, with remission rates for different EDs ranging between 30 and 60\% (e.g. [4]), and little is known about how to proceed when first-line treatments are ineffective. To improve outcomes, novel treatment alternatives are needed.

Neuroimaging techniques have improved our understanding of the neural substrates involved in EDs. Neurobiological models suggest that $\mathrm{AN}, \mathrm{BN}$ and BED have unique and overlapping neural features, including aberrant functioning of "bottom-up" sub-cortical mesolimbic and reward-related regions and/or in "top-down" prefrontal regions [5, 6]. Atypical functioning of these neural systems is associated with alterations in cognition, reward and emotion, and these may drive and maintain illness related behaviours. These models provide the rationale for investigating and using braindirected treatments [7].

Here, we provide an update on the literature relating to the use of neuromodulation in EDs in the last 3 to 5 years. Neuromodulation encompasses non-implantable and implantable procedures that can be used to inhibit, stimulate, modify or regulate nervous system functioning for the treatment of disease [8]. This includes techniques ranging 
from modern non-invasive neurostimulation approaches, such as repetitive transcranial magnetic stimulation (rTMS) and transcranial direct current stimulation (tDCS), to older techniques, such as electroconvulsive therapy (ECT), as well as surgical, invasive methods such as deep brain stimulation (DBS) and vagus nerve stimulation (VNS; see Table 1 for overview of each method). New evidence relating to each neuromodulation technique in EDs is reviewed below.

\section{rTMS}

Modern non-invasive brain stimulation techniques prominently include rTMS. In rTMS, a current is passed through an electromagnetic coil to induce an increase (high frequency; HF-rTMS) or decrease (low frequency; LF-rTMS) in cortical excitability in target brain regions [9]. Overall, rTMS is well-tolerated and safe [10]. Therapeutic applications of rTMS are being investigated across psychiatric disorders; however, its use is best established in depression where protocols have been incorporated into clinical guidelines [11].

rTMS in Anorexia Nervosa We recently completed the first sham-controlled RCT of high-frequency rTMS applied to the left-DLPFC in 34 cases of severe and enduring anorexia (SEAN; the TIARA trial [12•]). At 4 months post-randomisation, we saw medium to large between-group effect sizes in mood (e.g. DASS-21, total $d=0.9$ ) and quality of life (e.g. EQ$5 \mathrm{D}-5 \mathrm{~L}, d=0.52)$ and small between-group differences for BMI and ED symptoms, all favouring real rTMS.

In an open 18-month follow-up of this trial, mood improvements remained broadly stable in the real rTMS group and there was a "catch-up" in the sham group, as $10 / 12$ of these participants subsequently took the opportunity to receive real rTMS treatment [13•]. In regard to BMI change scores, by 18 months, there was a medium betweengroup effect, along with a higher rate of weight recovery in the real rTMS group compared to sham (BMI above $18.5 \mathrm{~kg}$ / $\mathrm{m}^{2}: 46 \%$ vs. $\left.9 \% ; \chi^{2}=3.67, p=.056\right)$, suggesting the mood effects of rTMS on AN precede those on BMI.

A notable finding from the participants' qualitative feedback at the end of TIARA [13•] was a growing flexibility and relaxation around eating and food choices following rTMS. This finding was supported by data from the Food Choice Task (see [14] for task methodology). In the real rTMS group, there was a decrease in self-controlled food choices post-treatment (vs. baseline), reflected in increased selection of tasty-unhealthy foods [15]. This could have arisen because rTMS induced functional changes in the DLPFC and associated neurocircuits: This is partially supported by the arterial spin labelling data obtained in the TIARA study [16]. Unlike previous rTMS studies (e.g. [17]), participants that received real rTMS showed no differences in cerebral blood flow (CBF) at the stimulation site (DLPFC); they did, however, show greater reductions in amygdala CBF, and this was associated with longer-term weight gain [16]. It is possible that lower CBF in the amygdala was associated with reduced fear around food, and this enabled the reported increased flexibility around food post-rTMS (thus resulting in longer-term weight gain). Studies of this potential mechanism underlying weight gain following rTMS are warranted.

The DLPFC is the most widely used target for rTMS in EDs, which may not be optimal for all recipients, e.g. a recent report of rTMS to the left-DLPFC in a case of AN and comorbid depression described no improvement in AN or mood symptoms [18]. As data emerge, new protocols are being developed and alternative stimulatory targets are being examined. For example, a Canadian group targeted the dorsomedial prefrontal cortex (DMPFC) in a case series $(n=19 ;[19])$ and reported significant improvements in core symptoms of AN (EDE global score; $p=.010$ ), anxiety (BAI; $p<.001$ ) and mood (BDI; $p=.041$ ) symptoms, from pre- to post-treatment. Their data showed that lower pre-treatment resting state functional connectivity from the DMPFC to the right frontal pole and left angular gyrus correlated with ED symptom improvement. The frontal pole is functionally coactive with the salience network regions (e.g. DMPFC), which have a role in cognitive and impulsive control [20]. Therefore, patients with weaker pre-treatment connectivity between these regions, who also showed greatest improvement in ED symptoms, may be those with reduced capacity for cognitive/impulse control and for whom DMPFC-rTMS may be particularly well-suited. To target different elements of AN (e.g. rigidity), Woodside et al. [19] highlight the potential value of stimulating the right orbitofrontal cortex, which has demonstrated potential for treating obsessive compulsive disorder (OCD) [21].

rTMS in BN and BED Several case studies/series (e.g. [22, 23]) have reported reductions in binge and/or purge episodes in BN following rTMS; however, two RCTs have not found significant differences between real or sham rTMS groups in binge-purge symptoms [24, 25]. An ancillary study to Gay et al. [25] reported on the neurocognitive outcomes [26]: This included inhibitory control (go/ no-go task; [27]), impulsivity (Barratt Impulsiveness Scale; [28]), decision-making (Iowa Gambling Task; [29]) and sustained attention (D2 test; [30]) from pre- to post-treatment. Between-group analyses revealed no significant differences in neurocognition post-treatment, but within-group analyses showed improvements in the go/no-go task $(p=.03)$ and the BIS cognitive impulsivity subscale $(p=.01)$ in the rTMS group, only. On the IGT, the intermediate net score significantly improved $(p=.002)$ and a significantly higher proportion of participants understood the task contingencies 


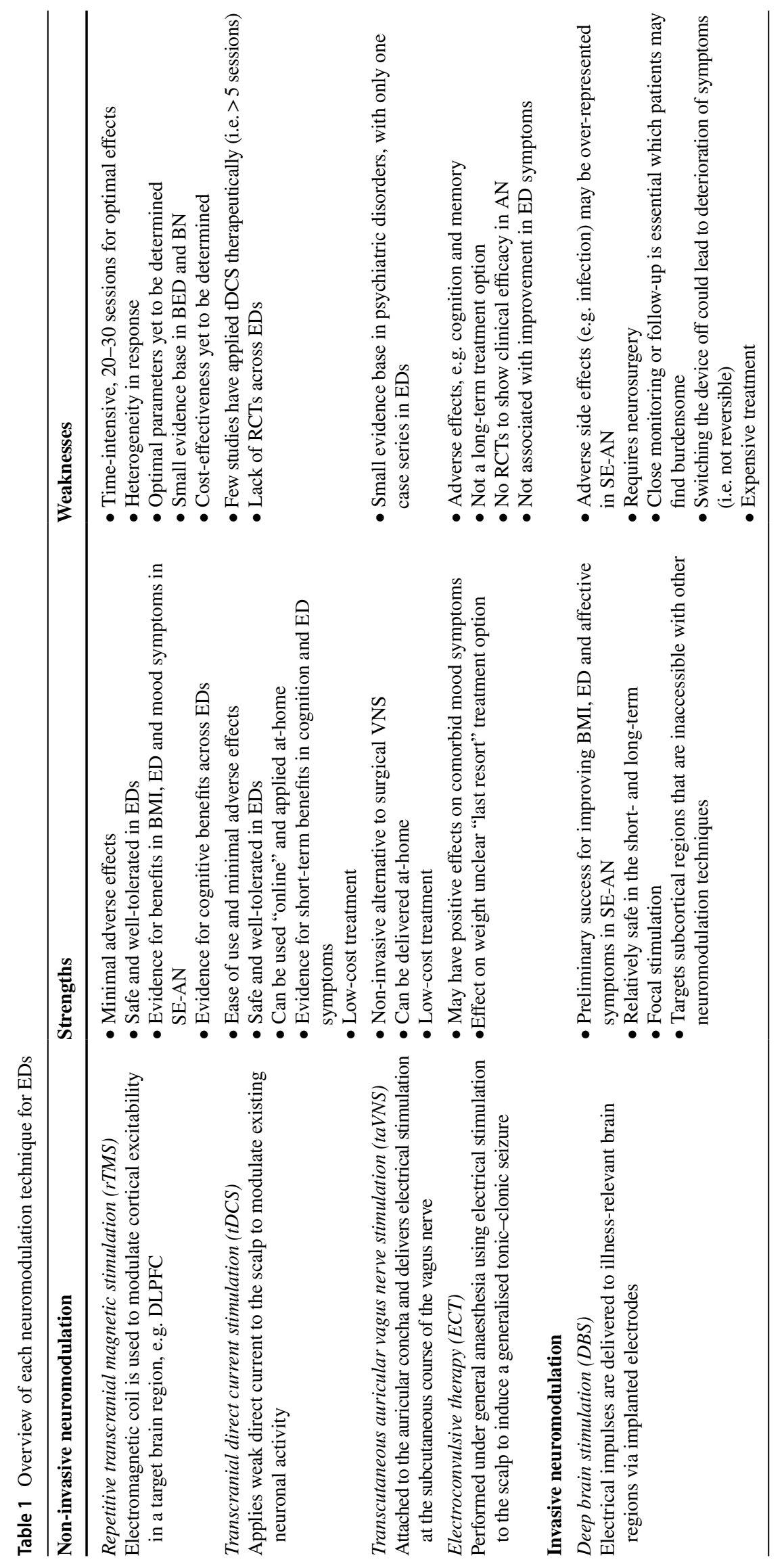


between pre- and post-treatment after real, but not sham, rTMS. These findings indicate that there are neurocognitive improvements following rTMS but that such changes are not necessarily sufficient to induce clinical improvements in $\mathrm{BN}[25,26]$.

Evidence for the use of rTMS in BED is lacking, although we anticipate the findings of a double-blind, sham-controlled RCT from Maranhão et al. [31].

\section{tDCS}

tDCS involves the application of a constant weak direct current via electrodes placed on the scalp to increase (anodal tDCS) or decrease (cathodal tDCS) cortical excitability [32]. Compared to other neuromodulation techniques, tDCS is easy to use, inexpensive and associated with fewer adverse effects [33, 34].

tDCS in Anorexia Nervosa To date, no RCT has investigated the use of tDCS in AN. A single-blind clinically controlled study in 23 adolescents with AN added either 18 sessions of DLPFC tDCS (anode left/cathode right) $(n=12)$, or family therapy $(n=11)$, to treatment as usual [35]. After 6 weeks of treatment, BMI increased in the tDCS group, an effect that persisted at 1-month follow-up. Participants in both groups showed similar improvements in AN psychopathology, mood and anxiety symptoms. Similarly, significant improvements in AN psychopathology, and a decrease in depression symptoms, were reported in a case series of ten inpatients with SE-AN following 20 sessions of anodal tDCS; however, no changes in BMI were observed [36].

A further case report applied $2 \mathrm{~mA}$ anodal tDCS to the left-DLPFC in a case of AN with comorbid PTSD [37]. Improvements were seen in self-reported body dissatisfaction and weight gain, but the onset of type 1 diabetes coincided with the use of tDCS. The effect of tDCS on glucose metabolism remains unclear; however, these findings are not consistent with reports that show reduced bloodglucose in healthy controls following repeated application of tDCS (e.g. [38]).

Finally, an ongoing pilot sham-controlled RCT in Australia [39] is using high-definition tDCS (i.e. more focal stimulation) applied to the left inferior parietal lobule (IPL) in participants with AN. The IPL is a novel neuromodulation target in AN and was selected due to evidence showing reduced connectivity in this region [40] and its role in eye movement, multi-sensory integration and body image. The findings will inform future trials targeting the IPL with tDCS in EDs.
tDCS in BN and BED A single session proof-of-concept RCT showed that $\mathrm{tDCS}$ may have potential for treatment of BN [41]. In adults with BED, a sham-controlled crossover trial [42-] investigated the effects of $1 \mathrm{~mA}(n=16)$ or $2 \mathrm{~mA}(n=15)$ anodal tDCS to the right-DLPFC, vs. sham, on response inhibition in a food-modified antisaccade task. Compared to sham, the 2-mA group improved in response inhibition, reflected in faster latencies of correct antisaccades, whereas the 1-mA group showed slower latencies. Only the 2-mA group showed a significant decrease in selfreported binge eating episodes [42•]. The nonlinear effects of tDCS, such that lower stimulation intensity (i.e. $1 \mathrm{~mA}$ ) was associated with significantly worse response inhibition, highlight the critical role for stimulation parameters in determining cognitive and/or clinical outcomes.

Our group has recently completed an RCT investigating the use of anodal tDCS to the right-DLPFC combined with approach bias modification (ABM) training for the treatment of adults with BED (see [43] for protocol). ABM training aims to retrain approach bias to appetitive cues into avoidance. Concurrently, administering tDCS is hypothesised to enhance neuroplasticity in pathways activated by the ABM and, in this way, is thought to amplify its efficacy. An integrated qualitative process evaluation [44] suggested that participants experienced ABM training and real/sham tDCS as an acceptable treatment combination, with few side effects; however, clinical outcomes are yet to be published.

An expanding area involves the use of at-home tDCS, which offers several advantages, including ease of access and increased compliance [45]. Several ongoing RCTs are assessing at-home tDCS as a treatment for BED. Our group is administering 10 sessions of concurrent at-home real/ sham tDCS and attention bias modification training [46], and Elkfury et al. [47] are combining at-home tDCS with a nutritional counselling therapy, delivering these separately or concurrently in a multi-arm RCT.

tDCS in Mixed ED Samples One study [48] examined the effects of tDCS on implicit preferences for food and body images across EDs (AN $n=21$; BN $n=13$; or ED not otherwise specified $n=2$ ). Their results showed that anodal tDCS to the medial prefrontal cortex, and the right extrastriate body area, modulated food preferences in EDs. This is relevant as implicit attitudes towards food are reported to predict maintenance of ED symptoms [49]. Future research should assess how these findings replicate in non-mixed ED samples.

\section{ECT}

ECT has long been used to treat mental illness, particularly when a rapid amelioration of severe and/or life-threatening 
symptoms is needed [50]. However, no randomised controlled trials (RCTs) have examined the efficacy of ECT in EDs. A recent systematic review and single case report by Pacilio et al. [51•] identified 14 cases with EDs treated with ECT from 11 publications. Case reports were mainly limited to AN, apart from one case of BED with comorbid obesity and bipolar disorder. Pre- and post-treatment BMI values were only reported in six cases. Of these, $50 \%$ of cases achieved a "normal" BMI over various follow-up periods, including the patient with BED who had a pre-treatment BMI of $97 \mathrm{~kg} / \mathrm{m}^{2}$ [51•]. The number of ECT sessions was highly variable across case reports (5-45), many patients had significant psychiatric comorbidities, two cases were older adults ( $>75$ years), and several cases were treated with ECT at a time when specialist ED treatments were not widely available. In their own report of a case with $\mathrm{AN}$ and recurrent depression and suicidal ideation, Pacilio et al. [51•] described improvements in mood following ECT, but no improvement in ED symptoms or BMI.

Since then, two further case reports have described the use of ECT in AN in the absence of comorbidities. One case showed gradual weight gain over 12 weeks following ECT [52]; the other case showed no improvement in ED, mood, or anxiety symptoms [53]. It is of note that in both reports where improvements in weight [52] or mood symptoms [51•] were reported, little to no change in attitudes towards weight, shape, or eating was observed. Thus, while the review and case studies available suggest potential value in using ECT to expedite weight restoration in AN [51•], they also highlight the need for adjunctive interventions that address weight and shape concerns. As such, given the evidence in favour of ECT is anecdotal and its safety and tolerability have been questioned, particularly due to the associated memory impairments, we would not presently recommend its use in EDs.

\section{DBS}

DBS is being investigated for its therapeutic potential in EDs, but, as it is an invasive procedure, its use has been limited to those with highly refractory SE-AN. DBS involves surgical implantation of electrodes into key structures (e.g. subcallosal cingulate, SCC [54]) implicated in ED pathology. In a recent randomised, sham-controlled, crossover trial [55•], DBS was applied to two different targets based on comorbidities: the SCC for affective disorders $(n=4)$ and nucleus accumbens (Nacc) for anxiety disorders $(n=4)$. This is relevant given the reported functional connectivity differences between "pure" EDs and EDs with comorbidities [56], as well as AN subtypes [57]. At 6-month followup (phase II), there were no significant changes in BMI from baseline to each month post-operatively. Villalba Martínez et al. [55•] surmised that as four participants underwent inpatient treatment to achieve the minimum BMI required for surgery $\left(13 \mathrm{~kg} / \mathrm{m}^{2}\right)$, the preoperative BMI values did not reflect their "usual" weight. Thus, a BMI reference value (BMI-RV) was calculated from mean BMI in the preoperative period. These findings showed a significant increase $(p=0.02)$ from BMI-RV to BMI at 6-month follow-up, with $5 / 8$ treatment responders $(\geq 10 \%$ increase in BMI$\mathrm{RV})$. Phase III of this trial is ongoing, with five responders randomised to one of two arms (DBS switched ON/ $\mathrm{OFF}$ or $\mathrm{OFF} / \mathrm{ON}$ ) and three non-responders not randomised and continuing with monthly assessments until 12-month follow-up.

The largest case series $(n=28)$ using DBS (to the $\mathrm{Nacc})$ in AN [58] reported significant increases in BMI at 6-month $(p<.001)$ and 2-year $(p<.001)$ follow-up, with (a) $43 \%$ of patients achieving a BMI $>18.5 \mathrm{~kg} / \mathrm{m}^{2} 2$ years post-operatively. In addition, obsessive-compulsive, mood and anxiety symptoms significantly decreased from baseline to 6-month and 2-year follow-up. Post hoc analysis revealed that mean BMI post-treatment was significantly lower in the binge-purge subgroup (AN-BP; $16.2 \mathrm{~kg} / \mathrm{m}^{2}$ ) than the restrictive subgroup (AN-R; $19.6 \mathrm{~kg} / \mathrm{m}^{2}$ ), which might mean that Nacc-DBS is less effective for weight restoration in AN-BP. Of note, the patients were described as "treatment-refractory" simply because many had BMIs $<15 \mathrm{~kg} / \mathrm{m}^{2}$ and all experienced amenorrhea [58]. However, their illness duration (mean 5.1 years; range 3-10) was markedly lower than other DBS studies in AN (e.g. [59•]) and it seems likely that some of these patients could have recovered with less invasive treatments.

Another group have recently published their $\geq 3$-year follow-up data from a case series $(n=22)$ using DBS to the SCC in SE-AN [59•]. Mean BMI increased from baseline $\left(14.0 \mathrm{~kg} / \mathrm{m}^{2}\right)$ to 1 -year $\left(p<0.001 ; 17.5 \mathrm{~kg} / \mathrm{m}^{2}\right)$ and 3 -year $\left(p<0.003 ; 16.3 \mathrm{~kg} / \mathrm{m}^{2}\right)$ follow-up. At the last followup ( $\geq 6$ years), $3 / 15$ patients reached BMI $\geq 18.5 \mathrm{~kg} / \mathrm{m}^{2}$ for $\geq 1$ year, and an additional 3/15 reached BMIs between 17 and $18.5 \mathrm{~kg} / \mathrm{m}^{2}$. After 1-year of SCC-DBS, there was also a significant improvement in obsessive-compulsive, depression and anxiety symptoms that was not maintained at 3-year follow-up. Of note, $20 \%$ of the participants experienced hardware infections and $27 \%$ had prolonged surgical site pain, which De Vloo et al. [59•] considered may be overrepresented in SE-AN cases compared to other DBS patients. Indeed, a further report [60] described hardware infection, as well as the re-emergence of symptoms, in a case of AN-BP who requested that the stimulation be turned off 19-months postoperatively. The key risk factors for infections following DBS implantation remain largely unclear [61]. Without this information, preventative measures cannot be implemented and the burden of infection (e.g. prolonged antibiotic therapy) may compromise the risk/benefit ratio of this treatment. 
Finally, DBS has been applied to the bed nucleus of the stria terminalis in a case of AN-R with significant improvements in weight and binge-purge symptoms [62]. The BNST is thought to be part of the extended amygdala and studies have increasingly used it as the stimulation target in OCD, with encouraging findings (e.g. [63]). Given that AN and OCD share some common neurobiological patterns (e.g. [64]), further examination of the BNST as a stimulation target for DBS in SE-AN seems appropriate.

\section{VNS}

Another invasive neuromodulation technique, VNS is an established treatment for several disorders (e.g. epilepsy), but it has yet to be used in EDs. However, transcutaneous auricular vagus nerve stimulation (taVNS) allows for noninvasive (i.e. without surgery) stimulation of the vagus nerve and is increasingly being studied in psychiatric disorders (see [65] for review). A recent study [66] applied 4 hours of taVNS per day over 9 weeks in a case series of mixed EDs (AN $(n=9), \mathrm{BN}(n=5), \mathrm{BED}(n=1))$. Results showed a significant reduction in depression and anxiety symptoms [66]. However, ED symptoms and BMI values were not reported. Therefore, further examination of this procedure, with appropriate and comprehensive clinical outcomes, is warranted.

\section{Conclusions}

In the present review, the majority of studies $(\sim 80 \%)$ have used neuromodulation in AN, with emphasis on rTMS and DBS. For example, findings from the TIARA RCT show that rTMS may be a promising treatment option for patients with SE-AN [12•, 13•]. However, despite encouraging results from rTMS to the DLPFC, outcomes remain heterogeneous, suggesting that DLPFC rTMS is by no means a panacea. Heterogeneity in outcomes may result from neuroanatomical, neurofunctional, connectivity-based, clinical and sociodemographic factors. Indeed, weaker DMPFC-frontal pole connectivity at baseline was associated with greater ED symptom improvement following rTMS to the DMPFC [20] and reductions in amygdala CBF following rTMS to the DLPFC were associated with long-term weight gain in SE-AN [16].

Ultimately, heterogeneity in response will be reduced when we move away from "one-size-fits-all" rTMS protocols for a given disorder and, instead, select optimal parameters based on individual biological, cognitive and clinical markers. This will require trials that incorporate multimodal neuroimaging, plus a range of neurocognitive tasks and clinical measures, as our group did in TIARA. Given the time commitment required, individualising patient care which uses reliable predictors is essential for avoiding arduous yet potentially futile courses of rTMS treatment [67]. Time demands may also be reduced by investigating the relative efficacy of theta burst stimulation (TBS) vs. standard rTMS in EDs, because findings show noninferiority of TBS in depression [68]. TBS is a newer variant of rTMS, but it offers significantly shorter daily stimulation sessions (<4 mins) compared to standard rTMS ( 40 mins).

Markedly fewer studies have examined neuromodulation use for BN and BED over the last 5 years. This may be related to negative findings in previous trials (e.g. Walpoth et al. [24]; Gay et al. [25]); however, these were small and had methodological limitations. We therefore encourage further investigation of neuromodulation in $\mathrm{BN}$.

Compared to other neuromodulation approaches, there have been fewer publications of tDCS use in EDs over recent years. Nevertheless, those included in this review paint a promising picture for the positive effects of tDCS on neurocognition, i.e. response inhibition and food preferences [42•, 48]. Cognitive dysfunctions are implicated in the development and maintenance of EDs (e.g. [69]), and thus, improvements in neurocognitive outcomes following tDCS are valuable and may be associated with clinical improvement. It will be of interest to see the outcomes from ongoing trials (e.g. [46, 47]), in particular the use of at-home tDCS in ED's.

The evidence from recent reports using DBS in SE-AN showed improvements in obsessive-compulsive, depression and anxiety symptoms and BMI, at 1-year [59•] and 2-year [58] follow-ups. However, these were not maintained at $\geq 6$-year follow-up in the De Vloo et al. [59•] case series. This may have been due to factors, such as (a) natural disease progression, (b) habituation or (c) placebo effect. If due to (a), this could explain why participants with a markedly lower mean illness duration (5.1 years) maintained significant decreases in clinical symptoms at 2-year follow-up in Liu et al. [58]. Habituation has been reported in other DBS indications (e.g. [70]) and can be mitigated through intermittent, rather than continuous, stimulation, i.e. closed-loop DBS, which was recently used for the first time in a psychiatric disorder [71]. In a case of treatment-resistant depression, Scangos et al. [71] identified amygdala gamma power as a biomarker of depression-specific symptoms for the patient. When prespecified patterns of amygdala gamma power were detected, this triggered $6 \mathrm{~s}$ of stimulation to the ventral capsule/ventral striatum. Within 12 days of stimulation, the patient's symptoms of depression and symptom severity had rapidly improved. Thus, closed-loop DBS presents a potentially exciting avenue for future studies in SE-AN.

Importantly, sham-controlled trials are a necessary next step to quantify the placebo effect of DBS in SE-AN. Though the inclusion of a sham-arm poses a moral/ethical challenge in a neurosurgical context, Villalba Martínez and colleagues [55•] have modelled one solution by using a cross-over sham-controlled design with responders from 
phase II having DBS turned ON/OFF in phase III. With only five of eight participants qualifying for phase III in this trial, this highlights how carefully patients are selected for trials using DBS. To maximise sample sizes, we recommend that multi-centre clinical trials are employed in the future.

Finally, ECT use has been limited to AN with variable results [51•], while in some cases, it appeared to facilitate weight gain in the short term; this was not true for all cases, and, overall, it did not improve ED symptoms. Given the availability of alternative non-invasive techniques that are not associated with adverse cognitive effects, ECT appears a relatively suboptimal form of therapeutic neuromodulation for treatment of SE-AN.

Overall, while the evidence base suggests that neuromodulation approaches have therapeutic promise in SE-AN and most techniques are safe and well tolerated, there are gaps in the knowledge base. These include questions on the use of neuromodulation treatments in EDs other than SE-AN and issues related to their use as adjunctive versus stand-alone treatments, the relative efficacy of different stimulation methods (e.g. tDCS vs. rTMS), variants (e.g. TBS), stimulation sites and parameters and, lastly, the mechanisms and duration of effects. As we better clarify the neural network disturbances underlying EDs, and how we can use neuromodulation techniques to rectify these, we expect to see increased efficacy.

Funding LG is supported by a PhD studentship from the National Institute of Health Research (NIHR) Mental Health Biomedical Research Centre (BRC) at South London and Maudsley NHS Foundation Trust (SLaM) and King's College London (KCL). MF is funded by KCL International Postgraduate Research Scholarship. US receives salary support from the NIHR BRC for Mental Health, SLaM and Institute of Psychiatry, Psychology and Neuroscience, KCL and is also supported by an NIHR Senior Investigator Award.

\section{Compliance with Ethical Standards}

Conflict of Interest The authors declare no competing interests.

Disclaimer The views expressed in this publication are those of the authors and not necessarily those of the National Health Service, the NIHR, or the UK Department of Health.

Open Access This article is licensed under a Creative Commons Attribution 4.0 International License, which permits use, sharing, adaptation, distribution and reproduction in any medium or format, as long as you give appropriate credit to the original author(s) and the source, provide a link to the Creative Commons licence, and indicate if changes were made. The images or other third party material in this article are included in the article's Creative Commons licence, unless indicated otherwise in a credit line to the material. If material is not included in the article's Creative Commons licence and your intended use is not permitted by statutory regulation or exceeds the permitted use, you will need to obtain permission directly from the copyright holder. To view a copy of this licence, visit http://creativecommons.org/licenses/by/4.0/.

\section{References}

Papers of particular interest, published recently, have been highlighted as:

- Of importance

1. American Psychological Association. Diagnostic and statistical manual of mental disorders (fifth edition). Arlington, VA: American Psychiatric Association. 2013.

2. Treasure J, Duarte T, Schmidt U. Eating disorders. Lancet. 2020. https://doi.org/10.1016/S0140-6736(20)30059-3.

3. National Institute for Health and Care Excellence. Eating disorders: recognition and treatment [NICE guideline no. 69]. 2020; https://www.nice.org.uk/guidance/ng69.

4. Linardon J, Wade TD. How many individuals achieve symptom abstinence following psychological treatments for bulimia nervosa? A meta-analytic review. Int J Eat Disord. 2018. https:// doi.org/10.1002/eat.22838.

5. Kessler RM, Hutson PH, Herman BK, Potenza MN. The neurobiological basis of binge-eating disorder. Neurosci Biobehav Rev. 2016. https://doi.org/10.1016/j.neubiorev.2016.01.013.

6. Steinglass JE, Berner LA, Attia E. Cognitive neuroscience of eating disorders. Psychiatric Clinics. 2019. https://doi.org/10. 1016/j.psc.2018.10.008.

7. Glashouwer KA, Brockmeyer T, Cardi V, Jansen A, Murray $\mathrm{SB}$, Blechert J, et al. Time to make a change: a call for more experimental research on key mechanisms in anorexia nervosa. Eur Eat Disord Rev. 2020. https://doi.org/10.1002/erv.2754.

8. Krames ES, Hunter Peckham P, Rezai A, Aboelsaad F. What Is Neuromodulation? In: Krames ES, Peckham PH, Rezai AR, editors. Neuromodulation. San Diego: Academic Press; 2009. p. 3-8.

9. Rossi S, Hallett M, Rossini PM, Pascual-Leone A. Safety, ethical considerations, and application guidelines for the use of transcranial magnetic stimulation in clinical practice and research. Clin Neurophysiol. 2009. https://doi.org/10.1016/j.clinph.2009.08.016.

10. Lefaucheur J-P, Aleman A, Baeken C, Benninger DH, Brunelin J, Di Lazzaro V, et al. Evidence-based guidelines on the therapeutic use of repetitive transcranial magnetic stimulation (rTMS): an update (2014-2018). Clin Neurophysiol. 2020. https://doi.org/10. 1016/j.clinph.2019.11.002.

11. National Institute for Health and Care Excellence. Repetitive transcranial magnetic stimulation for depression [Interventional Procedures Guidance No. 542]. 2015. https://www.nice.org.uk/ guidance/ipg542.

12.•Dalton B, Bartholdy S, McClelland J, Kekic M, Rennalls SJ, Werthmann J, et al. Randomised controlled feasibility trial of real versus sham repetitive transcranial magnetic stimulation treatment in adults with severe and enduring anorexia nervosa: the TIARA study. BMJ Open. 2018. https://doi.org/10. 1136/bmjopen-2018-021531. The first double-blind shamcontrolled randomised controlled trial of rTMS in AN. At 4 months post-randomisation, the authors reported large between-group differences for mood and modest changes in BMI and ED symptoms, all favouring rTMS.

13. Dalton B, Lewis YD, Bartholdy S, Kekic M, McClelland J, Campbell IC, et al. Repetitive transcranial magnetic stimulation treatment in severe, enduring anorexia nervosa: an open longer-term follow-up. Eur Eat Disord Rev. 2020. https://doi.org/ 10.1002/erv.2766. Open 18-month follow-up for the TIARA trial. Mood improvements were largely maintained in the real rTMS group at 18 months, and there a "catch up" effect was observed in the sham group, with most having received real rTMS treatment by this point. Between-group differences in 
BMI at 18-month follow-up indicate that mood improvements may precede change in BMI following rTMS treatment.

14. Steinglass J, Foerde K, Kostro K, Shohamy D, Walsh BT. Restrictive food intake as a choice: a paradigm for study. Int J Eat Disord. 2015. https://doi.org/10.1002/eat.22345.

15. Dalton B, Foerde K, Bartholdy S, McClelland J, Kekic M, Grycuk $\mathrm{L}$, et al. The effect of repetitive transcranial magnetic stimulation on food choice-related self-control in patients with severe, enduring anorexia nervosa. Int J Eat Disord. 2020. https://doi.org/10. 1002/eat.23267.

16. Dalton B, Maloney E, Rennalls SJ, Bartholdy S, Kekic M, McClelland J, et al. A pilot study exploring the effect of repetitive transcranial magnetic stimulation (rTMS) treatment on cerebral blood flow and its relation to clinical outcomes in severe enduring anorexia nervosa. J Eat Disord. 2021. https://doi.org/10.1186/ s40337-021-00420-w.

17. Kito S, Fujita K, Koga Y. Changes in regional cerebral blood flow after repetitive transcranial magnetic stimulation of the left dorsolateral prefrontal cortex in treatment-resistant depression. $\mathbf{J}$ Neuropsychiatry Clin Neurosci. 2008. https://doi.org/10.1176/jnp. 2008.20.1.74.

18. Jaššová $\mathrm{K}$, Albrecht J, Papežová $\mathrm{H}$, Anders M. Repetitive transcranial magnetic stimulation (rTMS) treatment of depression and anxiety in a patient with anorexia nervosa. Med Sci Monit. 2018. https://doi.org/10.12659/MSM.908250.

19. Woodside DB, Dunlop K, Sathi C, Lam E, McDonald B, Downar $\mathrm{J}$. A pilot trial of repetitive transcranial magnetic stimulation of the dorsomedial prefrontal cortex in anorexia nervosa: resting fMRI correlates of response. J Eat Disord. 2021. https://doi.org/10.1186/ s40337-021-00411-x.

20. Moayedi M, Salomons TV, Dunlop KAM, Downar J, Davis KD. Connectivity-based parcellation of the human frontal polar cortex. Brain Struct Funct. 2015. https://doi.org/10.1007/ s00429-014-0809-6.

21. Nauczyciel C, Drapier D. Stimulation magnétique transcrânienne répétée dans le traitement du trouble obsessionnel compulsif résistant. Revue Neurologique. 2012. https://doi.org/10.1016/j. neurol.2012.05.006.

22. Downar J, Sankar A, Giacobbe P, Woodside B, Colton P. Unanticipated rapid remission of refractory bulimia nervosa, during high-dose repetitive transcranial magnetic stimulation of the dorsomedial prefrontal cortex: a case report. Front Psychiatry. https:// doi.org/10.3389/fpsyt.2012.00030.

23. Dunlop K, Woodside B, Lam E, Olmsted M, Colton P, Giacobbe $\mathrm{P}$, et al. Increases in frontostriatal connectivity are associated with response to dorsomedial repetitive transcranial magnetic stimulation in refractory binge/purge behaviors. NeuroImage Clin. 2015. https://doi.org/10.1016/j.nicl.2015.06.008.

24. Walpoth M, Hoertnagl C, Mangweth-Matzek B, Kemmler G, Hinterhölzl J, Conca A, et al. Repetitive transcranial magnetic stimulation in bulimia nervosa: preliminary results of a singlecentre, randomised, double-blind, sham-controlled trial in female outpatients. Psychother Psychosom. 2008. https://doi.org/10.1159/ 000110061.

25. Gay A, Jaussent I, Sigaud T, Billard S, Attal J, Seneque M, et al. A lack of clinical effect of high-frequency rTMS to dorsolateral prefrontal cortex on bulimic symptoms: a randomised, double-blind trial. Eur Eat Disord Rev. 2016. https://doi.org/10.1002/erv.2475.

26. Guillaume S, Gay A, Jaussent I, Sigaud T, Billard S, Attal J, et al. Improving decision-making and cognitive impulse control in bulimia nervosa by rTMS: an ancillary randomized controlled study. Int J Eat Disord. 2018. https://doi.org/10.1002/eat.22942.

27. Schmitz N, Arkink EB, Mulder M, Rubia K, Admiraal-Behloul F, Schoonmann GG, et al. Frontal lobe structure and executive function in migraine patients. Neurosci Lett. 2008. https://doi. org/10.1016/j.neulet.2008.05.033.
28. Baylé FJ, Bourdel MC, Caci H, Gorwood P, Chignon J-M, Adés $\mathrm{J}$, et al. Structure factorielle de la traduction française de l'échelle d'impulsivité de Barratt (BIS-10). Can J Psychiatry. 2000. https:// doi.org/10.1177/070674370004500206.

29. Bechara A, Damasio H, Tranel D, Damasio AR. Deciding advantageously before knowing the advantageous strategy. Science. 1997. https://doi.org/10.1126/science.275.5304.1293.

30. Uttl B, Pilkenton-Taylor C. Letter cancellation performance across the adult life span. Clin Neuropsychol. 2001. https://doi. org/10.1076/clin.15.4.521.1881.

31. Maranhão MF, Estella NM, Cury MEG, Amigo VL, Picasso CM, Berberian A, et al. The effects of repetitive transcranial magnetic stimulation in obese females with binge eating disorder: a protocol for a double-blinded, randomized, sham-controlled trial. BMC Psychiatry. 2015. https://doi.org/10.1186/s12888-015-0569-8.

32. Nitsche MA, Paulus W. Transcranial direct current stimulation: update 2011. Restor Neurol Neurosci. 2011. https://doi.org/10. 3233/RNN-2011-0618.

33. Aparício LVM, Guarienti F, Razza LB, Carvalho AF, Fregni F, Brunoni AR. A systematic review on the acceptability and tolerability of transcranial direct current stimulation treatment in neuropsychiatry trials. Brain Stimul. 2016. https://doi.org/10.1016/j. brs.2016.05.004.

34. Brunoni AR, Amadera J, Berbel B, Volz MS, Rizzerio BG, Fregni F. A systematic review on reporting and assessment of adverse effects associated with transcranial direct current stimulation. Int J Neuropsychopharmacol. 2011. https://doi.org/10.1017/S1461145710001690.

35. Costanzo F, Menghini D, Maritato A, Castiglioni MC, Mereu A, Varuzza C, et al. New treatment perspectives in adolescents with anorexia nervosa: the efficacy of non-invasive brain-directed treatment. Front Behav Neurosci. 2018. https://doi.org/10.3389/fnbeh. 2018.00133.

36. Strumila R, Thiebaut S, Jaussent I, Seneque M, Attal J, Courtet $\mathrm{P}$, et al. Safety and efficacy of transcranial direct current stimulation (tDCS) in the treatment of anorexia nervosa. The open-label STAR study Brain Stimulation. 2019. https://doi.org/10.1016/j. brs.2019.06.017.

37. Mares T, Ceresnakova S, Albrecht J, Buday J, Klasova J, Horackova $\mathrm{K}$, et al. The onset of diabetes during transcranial direct current stimulation treatment of anorexia nervosa: a case report. Front Psych. 2020. https://doi.org/10.3389/fpsyt.2020.00040.

38. Kistenmacher A, Manneck S, Wardzinski EK, Martens JC, Gohla G, Melchert UH, et al. Persistent blood glucose reduction upon repeated transcranial electric stimulation in men. Brain Stimul. 2017. https://doi.org/10.1016/j.brs.2017.03.011.

39. Phillipou A, Kirkovski M, Castle DJ, Gurvich C, Abel LA, Miles $\mathrm{S}$, et al. High-definition transcranial direct current stimulation in anorexia nervosa: a pilot study. Int J Eat Disord. 2019. https://doi. org/10.1002/eat.23146.

40. Phillipou A, Abel LA, Castle DJ, Gurvich C, Hughes ME, Rossell SL. Midbrain dysfunction in anorexia nervosa. Psychiatry Res Neuroimaging. 2019. https://doi.org/10.1016/j.pscychresns.2018. 11.004.

41. Kekic M, McClelland J, Bartholdy S, Boysen E, Musiat P, Dalton $\mathrm{B}$, et al. Single-session transcranial direct current stimulation temporarily improves symptoms, mood, and self-regulatory control in bulimia nervosa: a randomised controlled trial. PLoS One. 2017. https://doi.org/10.1371/journal.pone.0167606.

42.• Max SM, Plewnia C, Zipfel S, Giel KE, Schag K. Combined antisaccade task and transcranial direct current stimulation to increase response inhibition in binge eating disorder. Eur Arch Psychiatry Clin Neurosci. 2021. https://doi.org/10.1007/s00406-020-01164-5. Randomised sham-controlled trial examining the effect of stimulation intensity (1 mA vs. $2 \mathrm{~mA}$ ) on outcomes from tDCS treatment in adults with binge eating disorder. Findings demonstrate the importance of precision in stimulation protocols. 
43. Gordon G, Brockmeyer T, Schmidt U, Campbell IC. Combining cognitive bias modification training (CBM) and transcranial direct current stimulation (tDCS) to treat binge eating disorder: study protocol of a randomised controlled feasibility trial. BMJ Open. 2019. https://doi.org/10.1136/bmjopen-2019-030023.

44. Gordon G, Williamson G, Gkofa V, Schmidt U, Brockmeyer T, Campbell I. Participants' experience of approach bias modification training with transcranial direct current stimulation as a combination treatment for binge eating disorder. Eur Eat Disord Rev. 2021. https://doi.org/10.1002/erv.2859.

45. Sandran N, Hillier S, Hordacre B. Strategies to implement and monitor in-home transcranial electrical stimulation in neurological and psychiatric patient populations: a systematic review. J Neuroeng Rehabil. 2019. https://doi.org/10.1186/s12984-019-0529-5.

46. Flynn M, Campbell I, Schmidt U. A feasibility study of transcranial direct current stimulation with attention bias modification training for binge eating disorder (clinical trial registration no. NCT04424745). 2020. https://clinicaltrials.gov/ct2/show/NCT04424745.

47. Elkfury J, da Conceição AL, Bandeira JS, Brietzke AP, Vieira GM, Luca PM, et al. Effect of home-based transcranial direct current stimulation associated with nutritional counseling therapy on clinical and electrophysiological measures in binge eating disorder: a study protocol for a blind, randomized, controlled clinical trial. Princip Pract Clin Res. 2020. https://doi.org/10.21801/ppcrj. 2020.64.1.

48. Mattavelli G, Gallucci A, Schiena G, D’Agostino A, Sassetti T, Bonora $S$, et al. Transcranial direct current stimulation modulates implicit attitudes towards food in eating disorders. Int J Eat Disord. 2019. https://doi.org/10.1002/eat.23046.

49. Khan S, Petróczi A. Stimulus-response compatibility tests of implicit preference for food and body image to identify people at risk for disordered eating: a validation study. Eat Behav. 2015. https://doi.org/10.1016/j.eatbeh.2014.10.015.

50. Folkerts HW. Elektrokrampftherapie. Nervenarzt. 2011. https:// doi.org/10.1007/s00115-010-3130-5.

51.• Pacilio RM, Livingston RK, Gordon MR. The use of electroconvulsive therapy in eating disorders: a systematic literature review and case report. J ECT. 2019. https://doi.org/10.1097/yct.000000000000599. A comprehensive review of electroconvulsive therapy in eating disorders.

52. Naguy A, Al-Tajali A, Alamiri B. An adolescent case of treatmentrefractory anorexia nervosa favorably responded to electroconvulsive therapy. J ECT. 2019. https://doi.org/10.1097/yct.0000000000000582.

53. Duriez P, Maatoug R, Verbe J. Failure of electroconvulsive therapy to improve anorexia nervosa in the absence of other psychiatric comorbidities: a case report. J ECT. 2020. https://doi.org/10. 1097/yct.0000000000000678.

54. Lipsman N, Lam E, Volpini M, Sutandar K, Twose R, Giacobbe $\mathrm{P}$, et al. Deep brain stimulation of the subcallosal cingulate for treatment-refractory anorexia nervosa: 1 year follow-up of an open-label trial. Lancet Psychiatry. 2017. https://doi.org/10.1016/ S2215-0366(17)30076-7.

55.• Villalba Martínez G, Justicia A, Salgado P, Ginés JM, Guardiola $\mathrm{R}$, Cedrón $\mathrm{C}$, et al. A randomized trial of deep brain stimulation to the subcallosal cingulate and nucleus accumbens in patients with treatment-refractory, chronic, and severe anorexia nervosa: initial results at 6 months of follow up. J Clin Med. 2020. https:// doi.org/10.3390/jcm9061946. A small cross-over DBS trial in AN which navigates the ethical/moral challenge posed by the inclusion of a sham-control group.

56. Molina-Ruiz RM, García-Saiz T, Looi JCL, Via Virgili E, Rincón Zamorano M, de Anta TL, et al. Neural mechanisms in eating behaviors: a pilot fMRI study of emotional processing. Psychiatry Investig. 2020. https://doi.org/10.30773/pi.2019.0038.

57. Van Autreve S, De. Baene W, Baeken C, van Heeringen K, Vancayseele N, Vervaet M. Differential neural correlates of set-shifting in the bingeing-purging and restrictive subtypes of anorexia nervosa: an fMRI study. Eur Eat Disord Rev. 2016. https://doi.org/10.1002/erv.2437.

58. Liu W, Zhan S, Li D, Lin Z, Zhang C, Wang T, et al. Deep brain stimulation of the nucleus accumbens for treatment-refractory anorexia nervosa: a long-term follow-up study. Brain Stimul. 2020. https://doi.org/10.1016/j.brs.2020.02.004.

59.• De Vloo P, Lam E, Elias GJB, Boutet A, Sutandar K, Giacobbe P, et al. Long-term follow-up of deep brain stimulation for anorexia nervosa. J Neurol Neurosurg Psychiatry. 2021. https://doi.org/10. 1136/jnnp-2020-325711. A long-term follow-up study reporting sustained improvements in BMI up to $\sim 6$ years post-operatively and the attenuation of initial improvements in mood 3 years post-operatively in a case series of 22 patients with AN. Reasons for the attenuation of psychological improvement are comprehensively explored.

60. Fernandes Arroteia I, Husch A, Baniasadi M, Hertel F. Impressive weight gain after deep brain stimulation of nucleus accumbens in treatment-resistant bulimic anorexia nervosa. BMJ Case Reports. 2020. https://doi.org/10.1136/bcr-2020-239316.

61. Abode-Iyamah KO, Chiang H-Y, Woodroffe RW, Park B, Jareczek FJ, Nagahama Y, et al. Deep brain stimulation hardware-related infections: 10-year experience at a single institution. J Neurosurg. 2019. https://doi.org/10.3171/2017.9.JNS1780.

62. Manuelli M, Franzini A, Galentino R, Bidone R, Dell'Osso B, Porta $\mathrm{M}$, et al. Changes in eating behavior after deep brain stimulation for anorexia nervosa. A case study. Eating and Weight Disorders - Studies on Anorexia, Bulimia and Obesity. 2020. https://doi.org/ 10.1007/s40519-019-00742-4.

63. Luyten L, Hendrickx S, Raymaekers S, Gabriëls L, Nuttin B. Electrical stimulation in the bed nucleus of the stria terminalis alleviates severe obsessive-compulsive disorder. Mol Psychiatry. 2016. https://doi.org/10.1038/mp.2015.124.

64. Marsh R, Maia TV, Peterson BS. Functional disturbances within frontostriatal circuits across multiple childhood psychopathologies. Am J Psychiatry. 2009. https://doi.org/10.1176/appi.ajp. 2009.08091354.

65. Cimpianu C-L, Strube W, Falkai P, Palm U, Hasan A. Vagus nerve stimulation in psychiatry: a systematic review of the available evidence. J Neural Transm. 2017. https://doi.org/10.1007/s00702-016-1642-2.

66. Melis Y, Apicella E, Macario M, Dozio E, Bentivoglio G, Mendolicchio L. Trans-auricular vagus nerve stimulation in the treatment of recovered patients affected by eating and feeding disorders and their comorbidities. Psychiatr Danub. 2020;32(Suppl 1):42-6.

67. Dunlop K. Transdiagnostic predictors and mechanisms of treatment response to repetitive transcranial magnetic stimulation of the dorsomedial prefrontal cortex: University of Toronto. 2018.

68. Voigt JD, Leuchter AF, Carpenter LL. Theta burst stimulation for the acute treatment of major depressive disorder: a systematic review and meta-analysis. Transl Psychiatry. 2021. https://doi.org/ 10.1038/s41398-021-01441-4.

69. Tamiya H, Ouchi A, Chen R, Miyazawa S, Akimoto Y, Kaneda Y, et al. Neurocognitive impairments are more severe in the bingeeating/purging anorexia nervosa subtype than in the restricting subtype. Front Psych. 2018. https://doi.org/10.3389/fpsyt.2018.00138.

70. Fasano A, Helmich RC. Tremor habituation to deep brain stimulation: underlying mechanisms and solutions. Mov Disord. 2019. https://doi.org/10.1002/mds.27821.

71. Scangos KW, Khambhati AN, Daly PM, Makhoul GS, Sugrue LP, Zamanian $\mathrm{H}$, et al. Closed-loop neuromodulation in an individual with treatment-resistant depression. Nat Med. 2021. https://doi. org/10.1038/s41591-021-01480-w.

Publisher's Note Springer Nature remains neutral with regard to jurisdictional claims in published maps and institutional affiliations. 